\title{
Reduced bias growth of pure-phase cubic boron nitride
}

\author{
Dmitri Litvinov and Roy Clarke \\ Harrison M. Randall Laboratory of Physics, University of Michigan, Ann Arbor, Michigan 48109-1120
}

(Received 8 May 1997; accepted for publication 4 August 1997)

\begin{abstract}
We report results on an improved growth process for cubic boron nitride (c-BN) films. The films are deposited on a dc-biased silicon substrate using ion-assisted sputtering. First, we grow a BN template layer at a bias voltage which maximizes the $\mathrm{sp}^{3}$ content. After this template layer attains a thickness of $\sim 500 \AA$, corresponding to the coalescence of the mosaiclike grain structure, we find that we can reduce the substrate bias to about $50 \%$ of its initial value while sustaining pure phase c-BN growth. The reduction in nitrogen ion energy results in a dramatic increase in the growth rate as well as significantly improved film quality. () 1997 American Institute of Physics. [S0003-6951(97)03540-7]
\end{abstract}

All of the physical deposition techniques for cubic boron nitride (c-BN) thin film growth involve energetic ion irradiation of the surface to initiate and sustain the formation the $\mathrm{sp}^{3}$-bonded cubic phase. ${ }^{1}$ Without ion irradiation, the hexagonal form of boron nitride (h-BN) is formed. Although seemingly unavoidable, energetic ion bombardment of the surface has some negative consequences. It reportedly causes the buildup of stress ${ }^{2,3}$ in the films and causes structural damage. Thus, eliminating ion irradiation or, at least, significantly decreasing the ion energies required to form the cubic phase, is a feasible way to improve film quality. ${ }^{4-6}$

In this work we describe a modification of the ionassisted sputter deposition technique developed by Kidner et al. ${ }^{7}$ that permits growth of pure-phase cubic boron nitride films at significantly reduced ion energies compared to those required in the initial nucleation and coalescence stages of the growth. This development results in a notable improvement in the structural quality of the films along with a substantial increase of growth rates.

We conducted the growth studies in a custom-designed ultrahigh vacuum (UHV) chamber with a base pressure of $1 \times 10^{-10}$ Torr. $^{8}$ Hot pressed boron nitride of $4 \mathrm{~N}$ purity was used as a sputtering target and nitrogen ions were supplied from an electron cyclotron resonance (ECR) source. The silicon (100) substrate, which was heated to over $1000{ }^{\circ} \mathrm{C}$ by direct Joule heating, was biased with a negative dc voltage to control the energy of nitrogen ions arriving at the film surface. At this temperature, the low base pressure of our vacuum chamber allowed thermal desorption of oxygen from the substrate surface, eliminating the need for any special surface treatment prior to deposition.

Reflection high energy electron diffraction (RHEED) was used as an in situ characterization of the film's surface structure, noting that h-BN and c-BN have very distinct RHEED patterns. In addition we used Fourier transform infrared spectroscopy (FTIR) to provide information about c-BN content, ${ }^{9,10}$ film stress, ${ }^{11}$ and average grain size. ${ }^{12}$ The vibrational frequencies of the infrared-active phonon modes for the cubic and hexagonal phases of BN are well known and tabulated. ${ }^{13}$ In particular, the h-BN phase has two modes at $770 \mathrm{~cm}^{-1}\left(\mathrm{~A}_{2 \mathrm{u}}\right.$ mode $)$ and $1383 \mathrm{~cm}^{-1}\left(\mathrm{E}_{1 \mathrm{u}}\right.$ mode $)$ and c-BN has a peak in its absorption spectrum at $\sim 1065 \mathrm{~cm}^{-1}$ corresponding to the TO phonon mode. ${ }^{14}$ The position of the c-BN peak depends strongly on the stress in the film ${ }^{11}$ while the width of the peak can provide qualitative information about grain size and structural defect density.

The c-BN thin film growth was initiated by setting the substrate bias (nitrogen ion energy) at a value that maximizes the cubic phase content as determined by FTIR. We found that a bias of $-96 \mathrm{~V}$ produced the largest $\mathrm{sp}^{3} / \mathrm{sp}^{2}$ ratio ( $\sim 90 \%$ ) for the deposition conditions selected here (nitrogen current density $\sim 1 \mathrm{~mA} / \mathrm{cm}^{2}$, substrate temperature $\mathrm{T} \approx 1000{ }^{\circ} \mathrm{C}$, sputtering gun power $\left.\sim 500 \mathrm{~W}\right)$. The initial phase of the growth (the "template layer') was carried out at this bias for $3 \mathrm{~h}$. Subsequent to the growth of the template layer, the bias voltage was reduced as described below, thus lowering the energy of the nitrogen ions incident on the surface of the growing film.

This $3 \mathrm{~h}$ growth period corresponds approximately to a minimum critical thickness of the c-BN template layer below which there is no sustained formation of the cubic phase with the reduced nitrogen ion energies as determined by RHEED and FTIR. The minimum thickness of the BN template layer coincides with the film thickness $(\sim 500 \AA)$ at which coalescence of the single crystal grains occurs to form a smooth surface layer. ${ }^{15}$ Thus a growth time of $\sim 3 \mathrm{~h}$ is necessary to obtain a film of sufficient thickness to complete the nucleation and coalescence process for the template layer. The growth of the BN template layer was followed immediately by growth at the reduced bias with all other deposition conditions kept fixed, unless otherwise specified. The FTIR absorption spectrum of the template layer on a silicon substrate was used as a background spectrum to obtain FTIR spectra of the films subsequently grown with the reduced substrate bias.

We have grown a series of c-BN films on $\mathrm{BN}$ template layers at different substrate bias voltages, growth times, and growth temperatures. Figure 1 shows a series of FTIR absorption spectra of the boron nitride films grown at different bias voltages. All of these films were grown for $2.5 \mathrm{~h}$ on top of the BN template layer described above. It can be clearly seen that there are no h-BN peaks down to a bias of $-56 \mathrm{~V}$ while the magnitude of the c-BN peak increases substantially at reduced bias. Since the area underneath the FTIR peak represents the amount of absorbing material, it is clear that the bias reduction leads to dramatically higher growth rates of the cubic phase (see inset). The reduction of bias voltage 


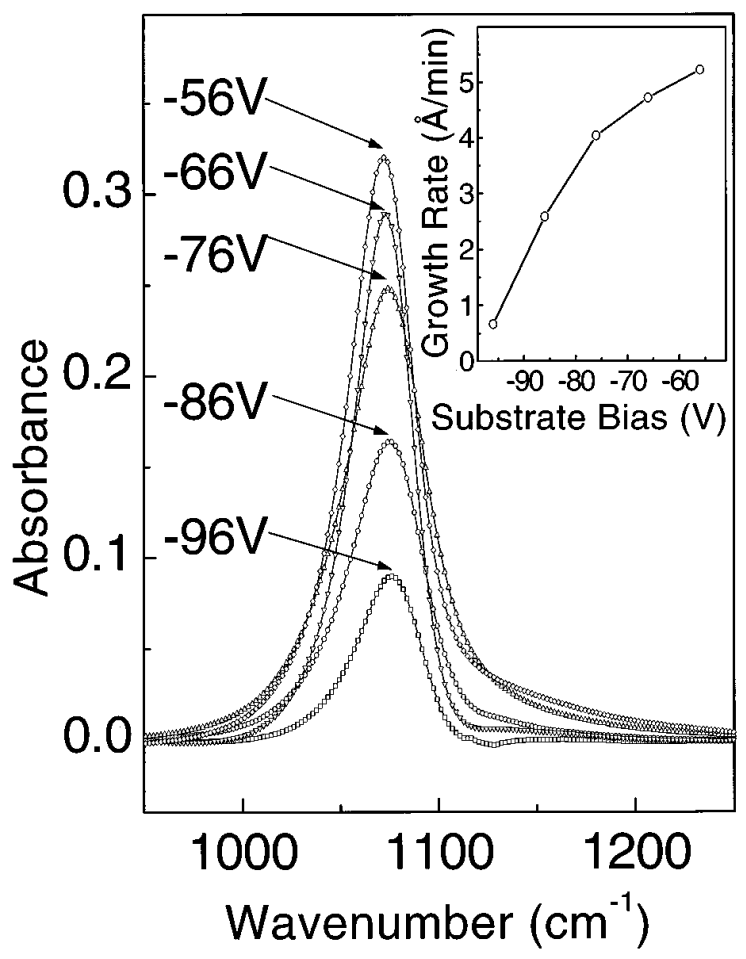

FIG. 1. FTIR absorption spectra for c-BN films grown at different substrate bias voltages.

below approximately $-50 \mathrm{~V}$ leads to the formation of predominantly hexagonal-phase material (h-BN).

Figure 2 presents the substrate bias dependence of the c-BN TO phonon frequency in Fig. 1. After nucleation and coalescence is complete, shifting of the peak center towards lower frequencies indicates a reduction of the strain in the film. ${ }^{16}$

Figure 3 compares the RHEED patterns of a film grown at $-96 \mathrm{~V}$ bias and a film grown at $-66 \mathrm{~V}$ bias. The RHEED

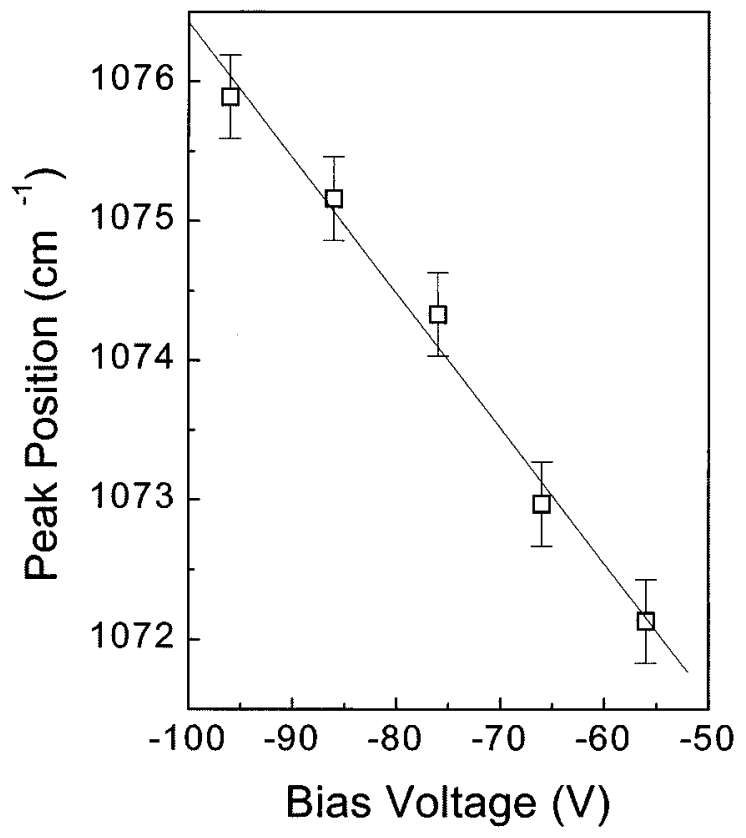

FIG. 2. c-BN TO-peak frequency vs substrate bias. The line is a guide to the eye.
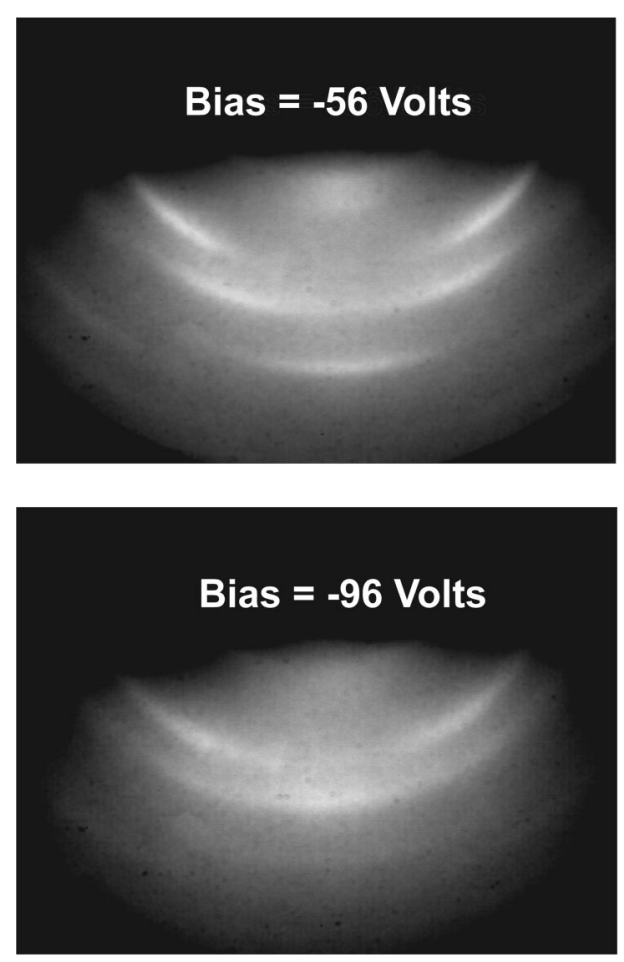

FIG. 3. RHEED patterns for c-BN films grown at different substrate bias voltages.

pattern of the film grown at the lower bias exhibits sharper diffraction arcs and shorter and better resolved higher order diffraction features than the RHEED pattern for the higher bias. These features indicate a higher degree of order within the film surface both in terms of long range positional order (i.e., large grains) as well as improved orientational ordering (i.e., mosaicity).

To make sure that the changes in c-BN FTIR peak width and peak position are not dominated purely by the change in the film thickness, we grew two films of the same thickness (different growth times were used to match the thickness of the films) at the different substrate bias conditions. Figure 4

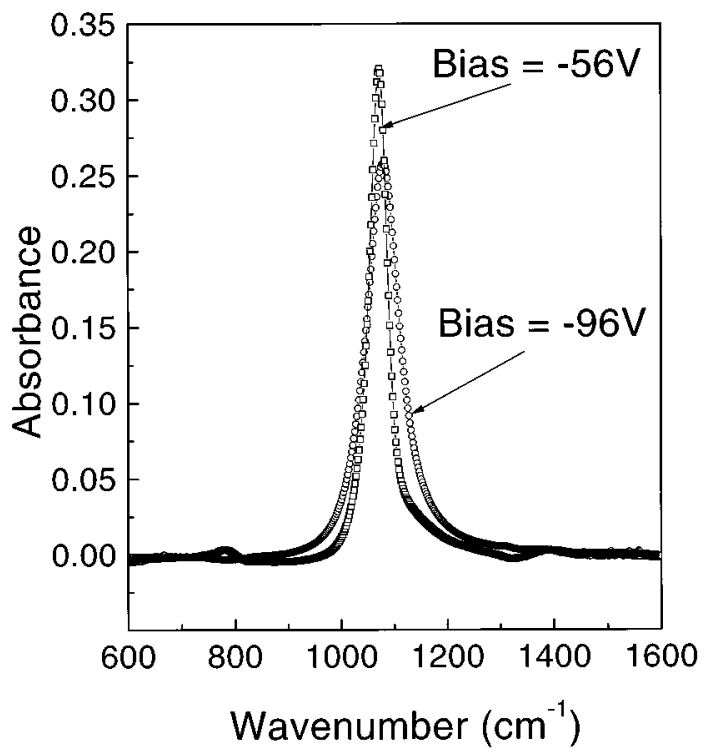

FIG. 4. FTIR absorption spectra of two c-BN films of the same thickness grown at different bias voltages. The peak at $-56 \mathrm{~V}(-96 \mathrm{~V})$ bias is centered on $1074 \mathrm{~cm}^{-1}\left(1080 \mathrm{~cm}^{-1}\right)$ and has a width of $48 \mathrm{~cm}^{-1}\left(88 \mathrm{~cm}^{-1}\right)$. 


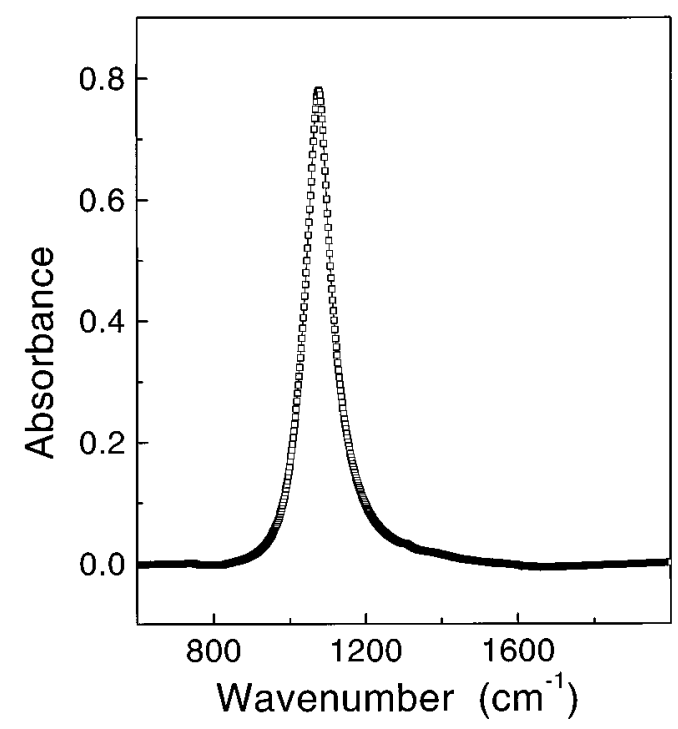

FIG. 5. FTIR absorption spectrum of a "thick" c-BN film grown at low bias $(-66 \mathrm{~V})$ for $8.5 \mathrm{~h}$.

compares the FTIR absorption spectra for two films of the same thickness grown at bias voltages of $-56 \mathrm{~V}$ and $-96 \mathrm{~V}$. The film grown with the lower substrate bias had less stress and higher film quality (larger grain size and less structural defects) as indicated by the c-BN peak position and peak width, respectively.

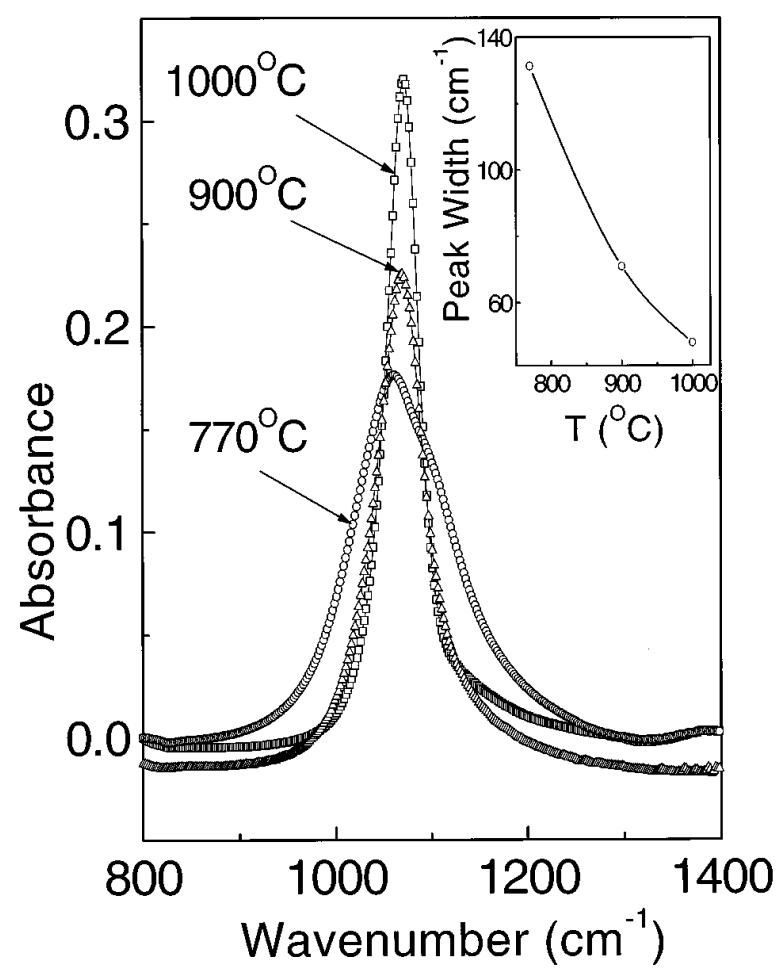

FIG. 6. FTIR absorption spectra of c-BN films grown at different temperatures.
We also grew a "thick" film at low bias $(-66 \mathrm{~V})$ for 8.5 $\mathrm{h}$ (corresponding to a thickness of $\sim 2500 \AA$ ) to check whether or not the growth of the c-BN phase would revert back to formation of the hexagonal phase with longer growth times. The FTIR absorption spectrum for this film (Fig. 5) shows no evidence of h-BN formation and is therefore purephase c-BN.

Figure 6 compares FTIR absorption spectra for several films grown at the same low bias $(-56 \mathrm{~V})$ but at different temperatures. It is evident that the growth temperature has a dramatic effect on the peak widths (see inset): higher growth temperatures promote higher quality film growth, confirming previously reported measurements. ${ }^{15}$

In conclusion, we have investigated the reduced bias growth of c-BN thin films on BN template layers corresponding to the completion of the nucleation and coalescence phases of growth. We have shown that the nitrogen ion energies necessary to initiate the formation of the cubic phase of boron nitride on Si (100) can be significantly decreased once a c-BN film of some minimum thickness is formed. The minimum thickness of the template layer is associated with the coalescence of nucleated grains to form a smooth surface layer. No stable epitaxial c-BN growth is observed below this thickness. The films grown with the reduced bias (lower energies of nitrogen ions) have less stress and fewer structural defects and the growth rate is also increased substantially.

This work is supported by ONR Grant Nos. N00014-91J-1398 and N00014-94-J-0763. The authors acknowledge useful discussions with S. Fahy.

${ }^{1}$ P. B. Mirkarimi, K. F. McCarty, and D. L. Medlin, Mater. Sci. Eng. Rept. (in press).

${ }^{2}$ D. R. McKenzie, W. D. McFall, W. G. Sainty, C. A. Davis, and R. E. Collins, Diam. Relat. Mater. 2, 970 (1993).

${ }^{3}$ P. B. Mirkarimi, K. F. McCarty, D. L. Medlin, W. G. Wolfer, T. A. Friedmann, and E. J. Klaus, J. Mater. Res. 9, 2925 (1994).

${ }^{4}$ D. R. McKenzie, W. D. McFall, H. Smith, B. Higgins, R. W. Boswell, A. Durandet, B. W. James, and I. S. Falconer, Nucl. Instrum. Methods Phys. Res. B 106, 90 (1995).

${ }^{5}$ S. Amagi, D. Takahashi, and T. Yoshida, Appl. Phys. Lett. 70, 946 (1997).

${ }^{6}$ P. B. Mirkarimi, D. L. Medlin, K. F. McCarty, D. C. Dibble, W. M. Clift, J. A. Knapp, and J. C. Barbour, J. Appl. Phys. (to be published, 1997).

${ }^{7}$ S. Kidner, C. A. Taylor II, and R. Clarke, Appl. Phys. Lett. 64, 1859 (1994).

${ }^{8}$ C. A. Taylor II, Ph.D. thesis, University of Michigan, Ann Arbor, 1996.

${ }^{9}$ D. J. Kester and R. Messier, J. Appl. Phys. 72, 504 (1992).

${ }^{10}$ S. Reinke, M. Kuhr, W. Kulisch, and R. Kassing, Diam. Relat. Mater. 4, 272 (1995).

${ }^{11}$ S. Fahy, Phys. Rev. B 51, 12873 (1995); 53, 11884 (1996).

${ }^{12}$ C. A. Taylor II and R. Clarke (unpublished).

${ }^{13}$ D. M. Hoffman, G. L. Doll, and P. C. Eklund, Phys. Rev. B 30, 6051 (1984).

${ }^{14}$ P. J. Gielisse, S. S. Mitra, J. N. Plendl, R. D. Griffis, L. C. Mansur, R. Marshall, and E. A. Pascoe, Phys. Rev. 155, 1039 (1967).

${ }^{15} \mathrm{C}$. A. Taylor II and R. Clarke, in NATO Advanced Research Workshop on Diamond, Diamond-related Materials, and Composites (Kluwer, New York, 1997).

${ }^{16}$ S. Fahy, C. A. Taylor II, and R. Clarke, Phys. Rev. B (in press). 